\title{
Metodología analítica y detección de residuos de ivermectina en muestras de leche de rebaños de la provincia de Nuble, Chile ${ }^{\#}$
}

\author{
Analytical methodology for the detection of ivermectin residues in milk \\ samples from dairy farms in the province of Nuble, Chile \\ L Pérez ${ }^{1}$, C Palma $^{1}$, R Villegas ${ }^{2}$, M Vega $^{2}$, R Pérez $^{1 *}$ \\ ${ }^{1}$ Laboratorio de Farmacología, Facultad de Medicina Veterinaria, Universidad de Concepción, Chillán, Chile. \\ ${ }^{2}$ Departamento de Bromatología, Facultad de Farmacia, Universidad de Concepción, Concepción, Chile.
}

\begin{abstract}
SUMMARY
A study was undertaken in order to validate a precise and reliable analytical method for ivermectin detection and quantification in milk and to determine the presence of ivermectin's residues in milk samples taken from dairy farms in the province of Ñuble. Analytical methodology was adequately validated using drug free milk samples fortified with increasing concentrations of ivermectin ( 0.5 to $25 \mathrm{ng} / \mathrm{mL}$ ), which were subjected to solid phase extraction and analyzed by high performance liquid chromatography. Milk samples were taken from the storage tanks of medium and high productive level dairy farms in the province of Nuble. Samples were collected in the four different season of the year: Summer (january), Fall (april), Winter (august) and Spring (November). The limit of quantification of the analytical method was established to be $0.043 \mathrm{ng} / \mathrm{mL}$. The absence of interferences and the adequate symmetry of the chromatograms suggest a good specificity of the method, with a recovery ranging from 73.5 to $79.5 \%$ for the minimal and maximal drug concentration, respectively. The analytical method presented satisfactory results of specificity, precision and accuracy that allow its use for the detection of residues of ivermectin in milk. Of 96 milk samples obtained from the storage tanks of dairy farms in the province of Nuble, $8.3 \%$ presented ivermectin concentrations which were greater than the limit of quantification of the analytical method. These results demonstrate that it is possible to find ivermectin residues in milk and they justify further studies in order to detect the presence of these drugs in milk, as their presence is a negative attribute in terms of quality.
\end{abstract}

Palabras clave: ivermectina, residuos, leche, HPLC.

Key words: ivermectin, residues, milk, HPLC.

\section{INTRODUCCION}

Para la mayoría de las explotaciones ganaderas el parasitismo gastrointestinal representa una amenaza permanente al estado sanitario del rebaño, debido a la contaminación continua de las praderas por huevos y larvas de nemátodos. A pesar de que en los animales de lechería la inmunidad les confiere una mayor resistencia, la intensificación de la producción puede aumentar la susceptibilidad al parasitismo. Estudios han demostrado que la inmunidad parasitaria puede disminuir durante los períodos de estrés aumentando la vulnerabilidad del ganado a la acción patógena de los parásitos. Estas interferencias con las defensas del hospedador pueden producir pérdidas de productividad como consecuencia de los efectos causados por la enfermedad subclínica (Hawkins 1993). Por lo tanto, el mejoramiento de la calidad pro-

Aceptado: 06.12.2005

\# Financiado por: Proyecto 202.151.012.-1.3 Dirección de Investigación Universidad de Concepción.

* Correspondencia debe ser dirigida a: Dr. Rubén Pérez F., Fax 5642 275302 rubperez@udec.cl Casilla 537, Chillán, Chile ductiva muchas veces obliga a la introducción de medidas en el manejo sanitario de la explotación que involucran el uso de antihelmínticos para el control del parasitismo. Además, se ha demostrado que el tratamiento antihelmíntico ejerce un efecto positivo sobre el aumento de la producción láctea en los animales tratados (Ploeger y col 1989)

En las últimas décadas el uso de ivermectina (IVM) se ha convertido en una de las alternativas de tratamiento de mayor eficacia y uso frecuente por parte de los ganaderos, debido a que se trata de un antihelmíntico de amplio espectro activo frente a formas adultas e inmaduras de nemátodos que afectan a los animales de producción (Campbell y col 1983, Shoop y col 1995).

La IVM es una lactona macrocíclica que deriva de productos de fermentación de la dihidroavermectina B1a, antihelmíntico que presenta una alta eficacia sobre endo y ectoparásitos de las diferentes especies animales. Su mecanismo de acción involucra tanto la potenciación de los efectos del ácido $\gamma$-amino butírico (GABA), un neurotransmisor inhibitorio de las respuestas motoras de los parásitos (Fischer y col 1993), como la interacción con canales glutamato-cloruro indepen- 
dientes de GABA, incrementando la permeabilidad de la membrana celular de las neuronas del parásito a los iones cloruro. De esta manera la ivermectina causa bloqueo neuromuscular, resultando en parálisis flácida y la eventual muerte del parásito (McKellar y Benchaoui 1996).

Las propiedades físico-químicas de la IVM incluyen un alto peso molecular y una elevada lipofilicidad, las que le confieren características farmacocinéticas de un alto volumen de distribución, con una gran afinidad por la grasa corporal y prolongada persistencia de sus concentraciones en el organismo (McKellar y Benchaoui 1996). La IVM se excreta principalmente por las heces. Sin embargo, en hembras en lactancia una fracción significativa del fármaco se excreta por la leche, en donde tiene una prolongada vida media (Alvinerie y col 1997).

Debido a sus características farmacocinéticas y al hecho de que una fracción muy significativa del fármaco se elimina a través de la leche (5\% de la dosis) su uso esta prohibido en animales en lactancia, cuyo producto sea destinado a consumo humano y se recomienda que su utilización en vacas gestantes se realice a lo menos con una anticipación de 28 días previos al parto (Alvinerie y col 1994).

Estudios realizados en vacas Frisonas productoras de leche tratadas con $0.2 \mathrm{mg} / \mathrm{kg}$ de IVM vía subcutánea, demostraron que la IVM es detectada en la leche a las 12 horas postadministración con una concentración máxima de $28,5 \mathrm{ng} / \mathrm{mL}$, mientras que en plasma esta fue de 41,2 ng/mL, estableciéndose una relación leche/plasma de 0,76 (Alvinerie y col 1997). La presencia de residuos en la leche permaneció durante un período de 17,8 días después del tratamiento (rango de 14-29 días). De igual modo, otros estudios determinaron que la concentración en el plasma de corderos lactantes, cuyas madres recibieron tratamiento con IVM, fue equivalente al $4 \%$ de la dosis de $0,2 \mathrm{mg} / \mathrm{kg}$ administrada por vía oral a las ovejas (Bogan y McKellar 1988).

La validación de un método analítico incluye todos los procedimientos requeridos para demostrar que una técnica analítica para la cuantificación de un determinado analito en una matriz biológica particular puede ser aplicada en forma segura y que sus resultados son confiables y representan las condiciones reales en las cuales se encuentra el compuesto (Shah y col 1992).

Diversos métodos analíticos han sido desarrollados para la detección y cuantificación de bajas concentraciones de IVM en diferentes matrices como plasma, tejidos y alimentos de consumo humano. La Cromatografía Líquida de Alta Resolución (HPLC), con extracción en fase sólida y formación de un derivado fluorescente por derivatización con $\mathrm{N}$-metilimidazol y anhídrido trifluoracético, ha permitido reducir significativamente el límite de detección de este fármaco y el tiempo de preparación de las muestras (De Montigny y col 1990, Alvinerie y col 1995).
El objetivo del presente trabajo fue validar un método sensible y confiable para la detección de ivermectina en leche y determinar la presencia de residuos de este antihelmíntico en muestras provenientes de predios lecheros de la provincia de Ñuble.

\section{MATERIALES Y METODOS}

MUESTRAS DE LECHE. Para el estudio se recolectaron muestras de leche provenientes de estanques de acumulación de 24 medianos productores lecheros de la provincia de Nuble, los cuales fueron muestreados en cuatro períodos del año; verano: enero, otoño: abril, invierno: agosto y primavera: noviembre. Estos predios tenían una producción de leche que fluctuaba entre los 800 y 3.800 L/día y el número de animales fluctuaba entre 50 y 220 vacas productoras. Las muestras fueron recolectadas directamente desde el estanque de acumulación de cada predio, con una capacidad promedio de 1.000 litros cada uno, al término de la ordeña de la mañana. Una vez homogeneizada la leche del estanque, la muestra fue depositada en un envase estéril de $100 \mathrm{~mL}$, en duplicado, las cuales se transportaron bajo condiciones de refrigeración, luego fueron almacenadas en cámara de congelación a $-20^{\circ} \mathrm{C}$, hasta su análisis. Se recolectaron 4 muestras por predio, lo que hace un total de 96 muestras.

METODOLOGIA ANALITICA. Se utilizó un cromatógrafo líquido de alta resolución* consistente en un sistema controlador, un módulo liberador de solventes, con unidad de gradiente de baja presión y desgasificador. El sistema, además, incluye un horno para columna y un detector espectrofluorométrico.

Reactivos. Los reactivos anhídrido trifluoracético y $\mathrm{N}$-metilimidazole, de grado analítico, fueron adquiridos de Sigma-Aldrich**. Los solventes acetonitrilo, metanol, ácido acético y agua grado HPLC fueron obtenidos de EM Science***. La IVM (principalmente dihidroavermectina $B_{1}$ a) fue obtenida de Sigma-Aldrich**

Soluciones estándar. A partir de una solución stock de $1,0 \mathrm{mg} / \mathrm{mL}$ en acetonitrilo se prepararon diluciones seriadas de concentraciones crecientes de $0,05,0,1,0,5$, 1 y $2,5 \mathrm{ng} / \mu \mathrm{L}$ de ivermectina para construir las curvas de calibración. Tanto la solución stock como las diluciones seriadas fueron diluidas en acetonitrilo y mantenidas a $4^{\circ} \mathrm{C}$, protegidas de la luz. Se preparó una solución de trabajo de $1 \mathrm{ng} / \mu \mathrm{L}$ de abamectina en metanol como estándar interno. En consideración a las propiedades de estabilidad en condiciones de congelación (Cerkvenick y col 2001), las soluciones stock de ivermectina y del

\footnotetext{
* Shimadzu, Kioto, Japón

** Sigma- Aldrich. Co, St. Louis, USA.

*** EM Industries, Inc. Gibbstown, New Jersey, USA.
} 
estándar interno abamectina, se prepararon cada tres meses mientras que las soluciones de trabajo fueron preparadas semanalmente.

Procedimientos analíticos. Las muestras de leche fueron sometidas a procesos de extracción y posterior derivatización, para luego ser analizadas por HPLC con detector de fluorescencia, después de la separación por fase sólida, de acuerdo al procedimiento descrito por Alvinerie y col (1993) y De Montigny y col (1990).

Extracción. Muestras de leche libre de fármaco $(1 \mathrm{~mL})$ fueron sobrecargadas con $10 \mu \mathrm{L}$ de estándar de IVM para alcanzar las siguientes concentraciones finales: 0,5, 1,0, $5,0,10,0,25,0 \mathrm{ng} / \mathrm{mL}$. Adicionalmente se le agregaron $10 \mathrm{uL}$ de solución de $1 \mathrm{ng} / \mu \mathrm{L}$ de abamectina como estándar interno, posteriormente fueron homogeneizadas por 30 segundos mediante el empleo de un agitador mecánico. De cada muestra se recogieron $500 \mu \mathrm{L}$ en duplicado y se depositaron en viales debidamente rotulados. Se adicionaron a cada vial $0,225 \mathrm{~mL}$ de agua $\left(4^{\circ} \mathrm{C}\right)$ y 0.5 $\mathrm{mL}$ de acetonitrilo $\left(4^{\circ} \mathrm{C}\right)$ y se llevó a agitador horizontal durante 20 min., posteriormente fueron sonicadas por 20 min. y luego centrifugadas a $13.000 \mathrm{rpm}$ por $10 \mathrm{~min}$. El total del sobrenadante de ambos viales, correspondientes a cada muestra de leche sobrecargada, fue transferido a un tubo de ensayo, al cual se adicionaron $5 \mathrm{~mL}$ de Hexano. Posteriormente se eliminó la fase orgánica y las muestras se centrifugaron a $5.000 \mathrm{rpm}$ por $5 \mathrm{~min}$., para luego ser sometidas a extracción en fase sólida mediante columnas de extracción (Supelclean LC18*) previamente acondicionadas con $2 \mathrm{~mL}$ de metanol y $2 \mathrm{~mL}$ de agua. Finalmente, la muestra se eluyó en 1,5 mL de metanol y el eluido se recolectó en tubos de ensayo.

Procedimientos de derivatización. El eluido $(0.750 \mathrm{~mL})$ se evaporó bajo flujo continuo de nitrógeno en un baño termorregulado a $60^{\circ} \mathrm{C}$ por $10 \mathrm{~min}$. El residuo seco fue disuelto en $100 \mu \mathrm{L}$ de una solución de $\mathrm{N}$-metilimidazol en acetonitrilo ( $1: 1 \mathrm{v} / \mathrm{v})$. Para iniciar la derivatización se adicionaron $150 \mu \mathrm{L}$ de una solución de anhídrido trifluoracético en acetonitrilo (1:2 v/v). Una vez terminada la reacción $(<30 \mathrm{~s}), 100 \mu \mathrm{L}$ de esta solución fueron inyectados directamente en el sistema cromatográfico por inyección normal (loop de $100 \mu \mathrm{L}$ ) mediante jeringa Hamilton.

Condiciones cromatográficas. La fase móvil constituida por ácido acético ( $0,2 \%$ en agua)-metanol-acetonitrilo $(2: 3464 \mathrm{v} / \mathrm{v} / \mathrm{v})$ fue bombeada a un flujo de $1,5 \mathrm{~mL} / \mathrm{min}$ a través de una columna Lichrospher RP18* (5 $\mu \mathrm{m} ; 4.6$ $\mathrm{mm} \times 150 \mathrm{~mm}$ ). Se empleó un detector de fluorescencia a longitud de onda de excitación de 383 nm y de emisión de $447 \mathrm{~nm}$.

\footnotetext{
* Supelco Inc., Bellefonte, PA, USA.
}

VALIDACION DEL METODO ANALITICO. La validación del método analítico tiene como objetivo demostrar que éste es adecuado para el propósito establecido y se realizó según los procedimientos descritos por Shah y col (1992), Codex Alimentarius (1995) y EMEA (1998) considerando los siguientes parámetros: especificidad, linealidad, porcentaje de recuperación, precisión y exactitud del método.

La especificidad del método se obtuvo comparando los resultados del análisis de sustancias presentes en las muestras con aquellas que contienen sólo el analito, determinando la capacidad del método para reconocer el analito en presencia de otros componentes de la muestra, como ser impurezas y el patrón interno.

La linealidad indica la capacidad del método para dar lugar a valores que son directamente proporcionales a la concentración del analito dentro del rango fijado y fue determinada mediante la inyección de muestras de leche sobrecargadas con IVM en concentraciones crecientes entre $0,5,1,0,5,0,10,0$ y $25 \mathrm{ng} / \mathrm{mL}$ a través de mediciones en quintuplicado. Las rectas de calibración fueron establecidas utilizando un análisis de regresión de mínimos cuadrados, representando las concentraciones de droga en el eje de la abscisa, y el área de los picos cromatograficos en el de la ordenada.

La recuperación del fármaco fue calculada comparando las áreas de los picos cromatográficos, en muestras de leche sobrecargadas con concentraciones conocidas de ivermectina, con las obtenidas de la inyección directa del estándar de IVM disuelto en metanol usando ABM como estándar interno. Los resultados se expresan en porcentajes (\%) y se obtuvieron de las muestras sobrecargadas con IVM en el rango de 0,5 a $25 \mathrm{ng} / \mathrm{mL}$.

La precisión, definida como la estimación de la variabilidad de las mediciones obtenidas de un muestreo múltiple y se expresa como el coeficiente de variación (C.V.) interdía e intradía de los resultados de la recta de calibración. La exactitud del método se definió como la diferencia porcentual entre el valor obtenido y el valor teórico, se expresó como el error medio (E.M., \%) para cada concentración de la recta de calibración de ivermectina.

Los límites de detección y de cuantificación se determinaron a partir de 10 inyecciones repetidas de muestras blanco libres de fármaco sobrecargadas con el estándar interno y se cuantificó el valor promedio del ruido en la línea base correspondiente al tiempo de retención de ivermectina (10,3 min). El promedio del ruido de la línea base más 3 desviaciones estándar se definió como el límite de detección. En tanto que el promedio del ruido de la línea base más 6 desviaciones estándar se estableció como el límite de cuantificación (Lifschitz y col 2000).

\section{ANÁLISIS ESTADISTICO}

Validación: El cálculo de los parámetros estadísticos: promedio, desviación estándar (DE), coeficiente de va- 
riación $(\mathrm{CV})$ y error medio o relativo (EM) fue realizado mediante el programa Microsoft Excel 97.

Muestras de predios lecheros: Se consideraron positivas a la presencia de residuos de IVM aquellas muestras que tuvieron una concentración igual o superior al límite de cuantificación del método.

\section{RESULTADOS}

\section{VALIDACION DEL METODO ANALITICO}

Especificidad. La capacidad del método de discriminar entre las impurezas de la leche y la presencia de los analitos en las muestras blanco sobrecargadas con IVM o el estándar interno (ABM) se muestran en las figuras 1A-B y C. Se aprecia una adecuada simetría de los picos cromatográficos para una concentración estándar de $10 \mathrm{ng} / \mathrm{mL}$ de IVM y $10 \mathrm{ng} / \mathrm{mL}$ de ABM como estándar interno. El tiempo de retención de IVM fue de 10,3 min., mientras que el de ABM como estándar interno fue de 7,8 min. La Figura 1B muestra el cromatograma correspondiente a una muestra de leche libre de fármaco. La ausencia de interferencias en los tiempos de retención de los analitos sugiere una alta especificidad del método. En la Figura 1C, se aprecia el cromatograma obtenido de una muestra de leche sobrecargado con $10 \mathrm{ng} / \mathrm{mL}$ de IVM y $10 \mathrm{ng} / \mathrm{mL}$ de ABM como estándar interno.

Linealidad. En el cuadro 1 se muestran los valores de los parámetros que definen la linealidad intradía e interdía. La ecuación de la recta de regresión que mejor describe la relación entre la concentración de fármaco y el área cromatográfica esta representada por: $\mathrm{y}=0,0399 \mathrm{x}+$

Cuadro 1. Linealidad intradía e interdía para muestras de leche bovina sobrecargadas con ivermectina en rangos de 0,5 a $25,0 \mathrm{ng} / \mathrm{mL}$, usando $10 \mathrm{ng} / \mathrm{mL}$ de abamectina como estándar interno.

Mean values of linearity for milk samples fortified with ivermectin at concentration ranging from 0.5 to $25 \mathrm{ng} / \mathrm{ml}$ and using abamectin as an internal standard.

\begin{tabular}{lccc}
\hline \multicolumn{1}{c}{$\mathrm{N}^{\circ}$ Muestra } & $\begin{array}{c}\text { Coeficiente de } \\
\text { correlación (r) }\end{array}$ & $\begin{array}{c}\text { Pendiente } \\
\text { (a) }\end{array}$ & $\begin{array}{c}\text { Ordenada en } \\
\text { el origen (b) }\end{array}$ \\
\hline Media & 0,99843 & 0,03990 & 0,00096 \\
$\mathrm{DE}$ & 0,00096 & 0,00131 & 0,00068 \\
$\mathrm{CV}(\%)$ & 0,09624 & 3,28 \\
& & & \\
& & INTERDIA & \\
Media & 0,99969 & 0,04449 & 0,029380 \\
$\mathrm{DE}$ & 0,00032 & 0,00077 & 0,001952 \\
$\mathrm{CV}(\%)$ & 0,03213 & 1,73 & - \\
\hline
\end{tabular}

0,0096, donde “ $x$ " representa la concentración de ivermectina $(\mathrm{ng} / \mathrm{mL})$, "a" la pendiente y "b" el intercepto cuyos promedios se presentan en el cuadro 1. El coeficiente de correlación fue siempre mayor que 0,990 en ambos parámetros. El coeficiente de variación fue de $1,73 \%$ (interdía) y de 3,28\% (intradía).

Recuperación. Los porcentajes de recuperación para cada punto considerado en la recta de calibración se presentan en el cuadro 2. El coeficiente de variación fluctuó entre $0,024 \%$ y $0,967 \%$, el rango de concentración entre 0,5 y $25 \mathrm{ng} / \mathrm{mL}$.

Precisión y exactitud del método. Los valores calculados de precisión y exactitud intradía e interdía expresados como los valores de coeficiente de variación (CV) y error medio (EM) para las diferencias encontradas entre el valor obtenido y el valor real, de la concentración de ivermectina en la curva estándar, se presentan en el cuadro 3 .

Limites de detección y de cuantificación. En el cuadro 4 se muestran las áreas cromatográficas y la relación de área entre las muestras blanco y las sobrecargadas con ABM como estándar interno utilizadas para la determinación de estas variables.

\section{RESIDUOS DE IVERMECTINA EN MUESTRAS DE PREDIOS LECHEROS DE LA PROVINCIA DE ÑULE}

En el cuadro 5 se muestran los resultados de los análisis de las muestras de leche provenientes de 24 predios de la provincia de Ñuble que fueron muestreados en 4 períodos del año. Como se observa del total de 96 muestras analizadas, $8(8,3 \%)$ señalaron concentraciones iguales o superiores al límite de detección del método analítico utilizado en el presente estudio.

Cuadro 2. Porcentajes de recuperación para las distintas concentraciones correspondientes a la curva de calibración de leche sobrecargada con ivermectina, usando $10 \mathrm{ng} / \mathrm{mL}$ de abamectina como estándar interno.

Percentage recovery for milk samples fortified with different concentrations of ivermectin and $10 \mathrm{ng} / \mathrm{ml}$ of abamectin as an internal standard.

\begin{tabular}{cccc}
\hline $\begin{array}{c}\text { Concentración de } \\
\text { IVM en muestra } \\
\begin{array}{c}\text { sobrecargada } \\
(\mathrm{ng} / \mathrm{mL})\end{array}\end{array}$ & $\begin{array}{c}\text { № } \\
\text { determinaciones }\end{array}$ & $\begin{array}{c}\text { Recuperación } \\
\text { (Prom. } \pm \mathrm{DE})\end{array}$ & CV \% \\
\hline 0,5 & 5 & $73,5 \pm 0,001$ & 0,024 \\
1,0 & 5 & $74,6 \pm 0,002$ & 0,041 \\
5,0 & 5 & $76,7 \pm 0,007$ & 0,198 \\
10,0 & 5 & $73,8 \pm 0,018$ & 0,392 \\
25,0 & 5 & $79,5 \pm 0,001$ & 0,967 \\
\hline
\end{tabular}


Cuadro 3. Precisión y exactitud intradía e interdía para muestras de leche bovina sobrecargadas con ivermectina en rangos de 0,5 a $25,0 \mathrm{ng} / \mathrm{mL}$, usando $10 \mathrm{ng} / \mathrm{mL}$ de abamectina como patrón interno.

Mean values of precision and accuracy for milk samples fortified with different concentrations of ivermectin and $10 \mathrm{ng} / \mathrm{ml}$ of abamectin as internal standard.

\begin{tabular}{ccccccccccc}
\hline & \multicolumn{3}{c}{ Concentración observada intradía } & & \multicolumn{3}{c}{ Concentración observada interdía } \\
$\begin{array}{c}\text { Concentración } \\
\text { Teórica }\end{array}$ & Promedio & DE & $\begin{array}{c}\text { CV } \\
\%\end{array}$ & $\begin{array}{c}\text { EM } \\
(\%)\end{array}$ & Promedio & DE & $\begin{array}{c}\text { CV } \\
\%\end{array}$ & $\begin{array}{c}\text { EM } \\
\%\end{array}$ \\
\hline 0,5 & 0,501 & 0,010 & 1,95 & 0,48 & & 0,49 & 0,012 & 0,02 & 2,00 \\
1,0 & 1,01 & 0,043 & 4,21 & 1,46 & 1,04 & 0,053 & 0,05 & 3,99 \\
5,0 & 4,90 & 0,058 & 1,19 & 2,03 & 4,87 & 0,292 & 0,06 & 2,61 \\
10,0 & 9,81 & 0,189 & 1,92 & 1,92 & 9,8 & 0,162 & 0,02 & 2,00 \\
25,0 & 25,73 & 0,313 & 1,22 & 2,83 & 25,1 & 0,232 & 0,01 & 0,40 \\
\hline
\end{tabular}

DE desviación estándar, CV coeficiente de variación, EM error medio.

Cuadro 4. Determinación del límite de cuantificación y límite de detección basado en la relación de área entre cromatográficas de muestras blancos $(\mathrm{Bk})$ y las áreas cromatográficas del estándar interno (ABM).

Determination of the detection and quantification limits based on the relationship signal-noise for blank milk samples fortified with $10 \mathrm{ng} / \mathrm{ml}$ of abamectin as an internal standard.

\begin{tabular}{|c|c|c|c|c|}
\hline $\begin{array}{l}\text { Muestra } \\
\text { Blanco }\end{array}$ & $\begin{array}{c}\text { Area } \\
\text { Blanco }\end{array}$ & $\begin{array}{l}\text { Area } \\
\text { ABM }\end{array}$ & $\begin{array}{c}\text { Relación } \\
\text { de Area } \\
\text { Blanco/ABM }\end{array}$ & $\begin{array}{c}\text { Concentración } \\
\text { calculada } \\
(\mathrm{ng} / \mathrm{mL})\end{array}$ \\
\hline 1 & 553 & 3104319 & 0,00018 & 0,031 \\
\hline 2 & 284 & 3059372 & 0,00009 & 0,034 \\
\hline 3 & 237 & 3044051 & 0,00008 & 0,033 \\
\hline 4 & 240 & 3046631 & 0,00008 & 0,034 \\
\hline 5 & 267 & 2940393 & 0,00009 & 0,033 \\
\hline 6 & 225 & 3015100 & 0,00007 & 0,029 \\
\hline 7 & 566 & 2999048 & 0,00019 & 0,030 \\
\hline 8 & 1703 & 2982537 & 0,00057 & 0,031 \\
\hline 9 & 1546 & 3001681 & 0,00052 & 0,029 \\
\hline 10 & 1710 & 2953715 & 0,00058 & 0,030 \\
\hline Media & & & 0,00024 & \\
\hline $\mathrm{DE}$ & & & 0,00022 & \\
\hline LC & & & & $0,0425 \mathrm{ng} / \mathrm{mL}$ \\
\hline LD & & & & $0,037 \mathrm{ng} / \mathrm{mL}$ \\
\hline
\end{tabular}

LC: límite de cuantificación (Media + 3DE).

LD: límite de detección (Media + 3DE).

\section{DISCUSION}

Los métodos analíticos utilizados para realizar determinaciones cuantitativas de fármacos y sus metabolitos en muestras biológicas juegan un importante rol en la evaluación e interpretación de los datos requeridos para el desarrollo de estudios de seguridad e inocuidad de alimentos destinados al consumo humano. Tales estudios son de fundamental importancia para la determinación de los límites máximos de residuos (LMR) y para esta-
Cuadro 5. Concentraciones de IVM (ng/mL) en muestras de leche bovina provenientes de predios de la provincia de Ñuble, muestreadas en 4 épocas durante el año.

Ivermectin concentrations in milk samples from dairy farms in the province of N Nuble sampled at four different times during the year.

\begin{tabular}{|c|c|c|c|c|}
\hline \multirow[b]{2}{*}{ Predio } & \multicolumn{4}{|c|}{ Epoca del año } \\
\hline & Primavera & Verano & Otoño & $\overline{\text { Invierno }}$ \\
\hline 1 & -* $^{*}$ & - & - & - \\
\hline 2 & 0,56 & - & - & 0,1 \\
\hline 3 & - & - & - & 0,05 \\
\hline 4 & - & - & - & - \\
\hline 5 & - & - & - & 0,81 \\
\hline 6 & - & - & - & - \\
\hline 7 & - & - & - & - \\
\hline 8 & - & - & - & 0,08 \\
\hline 9 & - & - & - & - \\
\hline 10 & - & - & - & - \\
\hline 11 & - & - & - & - \\
\hline 12 & - & - & - & - \\
\hline 13 & - & - & - & - \\
\hline 14 & - & - & - & - \\
\hline 15 & - & - & - & - \\
\hline 16 & 0,09 & - & - & - \\
\hline 17 & 0,22 & - & - & - \\
\hline 18 & - & - & - & - \\
\hline 19 & - & - & - & - \\
\hline 20 & - & - & - & - \\
\hline 21 & - & - & - & 0,92 \\
\hline 22 & - & - & - & - \\
\hline 23 & - & - & - & - \\
\hline 24 & - & - & - & - \\
\hline
\end{tabular}

*(-) Concentraciones bajo el límite de cuantificación del método.

blecer los periodos de carencia del fármaco. Por lo tanto, es esencial que estos métodos analíticos sean caracterizados y adecuadamente validados para obtener resultados que puedan ser satisfactoriamente interpretados. 
Existen diversos procedimientos analíticos para la detección de ivermectina mediante el empleo de cromatografía líquida (De Montigny y col 1990). Por lo general el uso de estas técnicas como método de detección implica una preparación complicada de la muestra, con la utilización de complejos compuestos que demandan gran cantidad de tiempo en su elaboración (Fischer y col 1993).

De acuerdo con los estudios de De Montigny y col (1990), Alvinerie y col (1993 y 1995), Fischer y col (1993) el uso de procedimientos de extracción en fase sólida para ivermectina resulta en la obtención de un extracto limpio con adecuados porcentajes de recuperación, hecho observado en el presente estudio, donde los porcentajes de recuperación para ivermectina fluctuaron entre 73,5 y 79,5\% para las diferentes concentraciones de droga utilizadas en la curva de calibración. Resultados similares, desde 74 a $81 \%$, han sido descritos por Alvinerie y col (1987) en muestras de leche fortificadas con concentraciones de ivermectina que fluctuaron entre los 20 y $100 \mathrm{ng} / \mathrm{mL}$. Al respecto el Codex Alimentarius (1995) indica que para muestras complejas como es la leche los valores de recuperación de un método analítico deben fluctuar entre 60 y $115 \%$.

El límite de cuantificación del método fue establecido en $0,04 \mathrm{ng} / \mathrm{mL}$ valor que es similar al descrito por Alvinerie y col (1977) de $0,05 \mathrm{ng} / \mathrm{mL}$ en leche de vaca.

La falta de interferencias y la adecuada simetría de los cromatogramas sugieren una buena especificidad del método, resultados que concuerdan con los descritos por De Montigny y col (1990) y Alvinerie y col (1995), quienes destacaron que el mejoramiento del proceso de derivatización de ivermectina, mediante el empleo de $\mathrm{N}$-metilimidazol y anhídrido trifluoroacético en acetonitrilo, permite una rápida formación (30 seg) del derivado fluorescente a temperatura ambiente $\left(25-30^{\circ} \mathrm{C}\right)$, disminuyendo los excesivos pasos de preparación de las muestras y los subproductos formados en la reacción que pueden afectar la sensibilidad del método.

La linealidad del procedimiento analítico (interdía e intradía), determinada por el análisis de regresión de la recta de calibración presentó coeficientes de correlación superiores a 0,990 , con un coeficiente de variación de $0,1 \%$. El valor de los CV calculados sugieren que la repetibilidad de la linealidad tanto interdía como intradía, es aceptable y que, por lo tanto, permitirá la utilización de las rectas de calibración en el rango establecido para la interpolación de valores relativos a concentraciones de ivermectina en muestras de leche bovina. De igual forma la precisión y exactitud (interdía e intradía) con la cual se determina la concentración de analito, expresadas a través del coeficiente de variación y el error relativo medio respectivamente, presentaron valores inferiores a los descritos por Shah y col (1992) como aceptables para la adecuada validación de un procedimiento analítico.
Los resultados obtenidos mediante el procedimiento de validación descrito, aseguran que un método preciso y confiable para la detección y cuantificación de residuos de ivermectina en leche a concentraciones mínimas de $0,042 \mathrm{ng} / \mathrm{mL}$ está disponible para la agencias encargadas de la regulación de residuos en alimentos y la industria lechera que les puede permitir detectar niveles muy bajos de estos fármacos. Este es un factor importante de considerar por tratarse de una molécula muy estable que resiste las condiciones de refrigeración, pasteurización y de cocción a $100^{\circ} \mathrm{C}$, ya sea de la leche (Cervenik y col 2001) o la carne (Slanina y col 1989). Además de que por su alta lipofilicidad se concentra en la materia grasa, lo que determina una mayor concentración en los productos lácteos como mantequilla, yogurt, crema y queso (Cervenik y col 2004).

\section{RESIDUOS DE IVERMECTINA EN PREDIOS LECHEROS DE LA PROVINCIA DE ÑUBLE}

Utilizando la metodología descrita, se determinó que el 8,3\% de las muestras provenientes de los estanques de recolección de los predios lecheros de la provincia de Nuble presentaron concentraciones de IVM superiores al limite de cuantificación del método analítico utilizado. Es importante destacar la adecuada sensibilidad del método para detectar concentraciones muy pequeñas del fármaco que pueden ser consideradas como trazas. Más aún si se considera el efecto de dilución que se produce al mezclar en un estanque leche de diferentes animales del mismo predio.

Las bajas concentraciones detectadas (rango de 0,05$0,92 \mathrm{ng} / \mathrm{mL}$ ) permiten pensar que el origen de los residuos proviene de sólo algunos animales y no del rebaño completo, producto probablemente del tratamiento selectivo de aquellos en los cuales se consideró necesario realizar una desparasitación. Es conocido que en aquellos predios lecheros en los cuales los animales están expuestos al parasitismo, ya sea porque se someten a pastoreo, o bien, porque se incorporan al rebaño en producción, como es el caso de las vacas de primer parto, el tratamiento antiparasitario se realiza durante el período seco previo al parto. A pesar de la indicación del laboratorio de no administrar en animales en lactancia ni en aquellas vacas gestantes que se encuentren dentro de los 60 días previo al parto, existe la posibilidad de que algunos ganaderos no respeten estos períodos de resguardo y se origine la presencia de residuos como se ha demostrado con los resultados del presente estudio. Se ha reportado que la ivermectina se puede detectar en la leche hasta 59 días después de tratar vacas lecheras durante el período seco previo al parto (Alvinerie y col 1994).

Es conveniente destacar que en el presente estudio las muestras positivas se detectaron durante los meses de invierno y primavera que pueden coincidir con la incorporación de vacas recién paridas al rebaño en 
producción lo que permite la posibilidad de que contengan residuos de ivermectina producto del tratamiento realizado durante el final de la gestación.

La presencia de residuos de IVM en la leche representa un aspecto que debe ser considerado desde el punto de vista de la salud pública por tratarse de un alimento de consumo masivo por la población. Especialmente si se considera que el fármaco es bien absorbido luego de la administración oral (Campbell y Benz 1984), es realista asumir que si los residuos están presentes, estos se encuentran disponibles para que sean ingeridos por el consumidor y en particular niños lactantes que se alimentan de leche materna o con leche de vaca (Toutain y col 1988). Sin embargo, es conveniente destacar que las concentraciones detectadas son tan bajas, que es poco probable que su ingestión represente un riesgo para la salud del consumidor.

Desde el punto de vista toxicológico, IVM es un fármaco seguro para los mamíferos adultos en consideración a que debe atravesar la barrera hematoencefálica (BHE) para actuar sobre neuronas en las cuales el GABA representa su principal neurotransmisor (Campbell y Benz 1984). Es conocido que este fármaco no atraviesa la BHE, de lo cual deriva su amplio margen de seguridad en mamíferos. Su gran tamaño molecular y el alto grado de unión a las proteínas del plasma limita su paso hacia el SNC a través de la BHE (Fisher y Mrozik 1992, Paul y Bajean 1993). Esta limitada distribución hacia el SNC parece ser el factor más importante en la diferenciación de la toxicidad selectiva hacia los invertebrados y de su amplio margen de seguridad para el huésped mamífero vertebrado (Campbell y Benz 1984, Bennet 1986). Sin embargo, algunos factores como el insuficiente desarrollo de la BHE en los recién nacidos o la acción de procesos febriles pueden determinar que se incremente el paso de moléculas de IVM hacia el SNC y logre concentraciones que sean capaces de actuar sobre las neuronas gabaergicas y ejercer alguna acción farmacológica.

Estudios experimentales en ratas han demostrado una mayor susceptibilidad a la acción de tóxica de ivermectina en animales recién nacidos y hasta los 10 días de edad, al ingerir leche de hembras lactantes tratadas con alta dosis del fármaco. Al parecer, esta mayor susceptibilidad de los recién nacidos está asociada al desarrollo incompleto de la BHE (Paul y Abjean 1993).

Los resultados del presente estudio demuestran la presencia de residuos de IVM en leche provenientes de predios cuya producción se destina a uso industrial para posterior consumo humano. Considerando que las agencias internacionales y el Codex Alimentarius establecen que la presencia de residuos de IVM en leche destinada a consumo humano no está permitida, es importante considerar la realización de estudios más amplios tendientes a evaluar esta situación con el fin de conocer la real magnitud del problema a nivel nacional.
Los resultados permiten concluir que el método analítico presenta resultados de sensibilidad, precisión y exactitud satisfactorios que permiten obtener resultados seguros y confiables en la detección de residuos de IVM en leche. Además los resultados de las muestras de leche provenientes de los estanques de recolección demuestran la posibilidad de que en algunos predios lecheros de la provincia de Nuble se utilice IVM para el control del parasitismo, por lo que es posible determinar la presencia de residuos y justifica la realización de estudios más amplios tendientes a evaluar la frecuencia de estos fármacos en la leche, debido a que su presencia es un atributo negativo de calidad.

\section{RESUMEN}

Se realizó un estudio con el objetivo de validar un método sensible y confiable para la detección de ivermectina en leche y determinar la presencia de residuos en muestras provenientes de predios lecheros de la provincia de Nuble. La validación de la metodología analítica se realizó en muestras de leche libres de fármaco sobrecargadas con ivermectina (IVM) $(0,5,1,0$, $5,0,10,0,25,0 \mathrm{ng} / \mathrm{mL})$, las que fueron sometidas a extracción en fase sólida y analizadas por cromatografía líquida de alta eficiencia (HPLC). Para el estudio de residuos se recolectaron muestras de leche desde estanques de acumulación de 24 predios pertenecientes a medianos productores de la provincia de Ñuble, en cuatro períodos del año: verano: enero, otoño: abril, invierno: agosto y primavera: noviembre. El límite de cuantificación del método fue establecido en 0,043 ng/mL. La ausencia de interferencias y la adecuada simetría de los cromatogramas sugieren una buena especificidad del método, con porcentajes de recuperación que fluctúan entre el 73,5 y $79,5 \%$, para la concentración mínima y máxima del fármaco. El método analítico presentó resultados de sensibilidad, precisión y exactitud satisfactorios que permiten su utilización en la detección de residuos de ivermectina en leche. De 96 muestras de leche obtenidas desde estanques de recolección de predios lecheros de la provincia de Ñuble, el 8,3\% presentó concentraciones de ivermectina iguales o superiores al límite de cuantificación del método analítico utilizado. Estos resultados demuestran la presencia de residuos de ivermectina en leche y justifican la realización de estudios más amplios tendientes a detectar estos fármacos en la leche, debido a que su presencia es un atributo negativo de calidad.

\section{REFERENCIAS}

Alvinerie M, JF Sutra, P Galtier, PL Toutain. 1987. Determination of ivermectin in milk by high performance liquid chromatography. Ann Rech Vet 18, 269-274.

Alvinerie M, JF Sutra, P Galtier, PL Toutain. 1993. Cinétiques plasmatiques de l'ivermectine chez la vache. Recueil Med Vet 169, 259-261.

Alvinerie M, JF Sutra, P Galtier, PL Toutain. 1994. Microdose d'ivermectine chez la vache laitière: concentrations plasmatiques et residus dan le lait. Revue Med Vet 169, 259-261.

Alvinerie M, JF Sutra, M Badri, P Galtier. 1995. Determination of moxidectin in plasma by high-performance liquid 
chromatography with automated solid-phase extraction and fluorescence detection. J Chromat B 674, 119 - 124.

Alvinerie M, JF Sutra, PL Toutain. 1997. Residus d'ivermectine dans le lait chez la vache laitiére traitée pendant la période de tarissement avec la posologie recommandée par l'autorisation de mise sur le marché. Revue Méd Vét 148, 115-116.

Bennet DG. 1986. Clinical pharmacology of ivermectin. JAVMA 189, 100-103.

Bogan JA, QA McKellar. 1988. The pharmacodynamics of ivermectin in sheep and cattle. $J$ Vet Pharmacol Therap 11, 260-268.

Campbell WC, GW Benz. 1984. Ivermectin: A review of efficacy and safety. $J$ Vet Pharmacol Ther 7, 1-16.

Campbell WC, M Fisher, E Stapley, G Albers-Schönberg, T Jacob. 1983. Ivermectin: A potent new antiparasitic agent. Science 221, 823-828.

Cerkvenik V, DZ Doganoc, V Skubic, WMJ Beek, HJ Keukens. 2001. Termal and long-term freezing stability of ivermectin residues in sheep milk. Eur Food Res Technol 213, 72-76.

Cerkvenik V, B Perko, I Rogelj, DZ Doganoc, V Skubic, WMJ Beek, HJ Keukens. 2004. Fate of ivermectin residues in ewes' milk and derived products. J Dairy Res 71, 39-45.

Codex Alimentarius, 1995. Residuos de Medicamentos Veterinarios. Vol. 3, 60-76.

De Montigny P, JK Shim, JV Pivinichny. 1990. Liquid chromatographic determination of ivermectin in animal plasma with trifluoroacetic anhydride and N-methylimidazole as the derivatization reagent. J Pharm \& Biom Anal 8, 507-511.

EMEA. 1998. Guideline on validation of analytical procedures: Methodology. VICH topic GL2: The European Agency for the Evaluation of Medicinal Products. Veterinary Evaluation Unit. London.

Fisher MH, H Mrozik. 1992. The chemistry and pharmacology of avermectins. Ann Rev Pharmacol Toxicol 32 , 537-553.
Fischer J, MT Kelly, MR Smyth, P Jandera. 1993. Determination of ivermectin in bovine plasma by columnswitching LC using on-line solid-phase extraction and trace enrichment. J Pharm \& Biom Anal 11, 217-223.

Hawkins JA 1993. Economic benefits of parasite control in cattle. Vet Parasitol 46, 159-173.

Lifschitz A, F Imperiale, G Virkel, M Muñoz, N Scherling, R Delay, C Lanusse. 2000. Depletion of Moxidectin Tissue Residues in Sheep. J Agric Food Chem 48, 6011-6015.

McKellar QA, H Benchaoui. 1996. Avermectins and milbemycins. $J$ Vet Pharmacol Therap 19, 331-351.

Paul JM, JP Abjean. 1993. Toxicité de l'ivermectine chez la rat nouveaunée. Rec Méd Vét 169, 47-52.

Payne LD, B Hicks, TA Wehner. 1995. Determination of abamectin and/or ivermectin in cattle feces at low parts per billion levels using HPLC with fluorescence detection. J Agric Food Chem 43, 1233-1237.

Ploegger HW, GJW Shoenmaker, A Klosterman, FH Borgsteede. 1989. Efecct of anthelmintic treatment of dairy cattle on milk production related to some parameters estimating nematode infection. Vet Parasitol 34, 239-253.

Shah VP, KK Midha, S Dighe, IJ Mcgilveray, JP Skelly, A Yacobi, T Layloff, C Viswanathan, C Edgar, R Mcdowall, KA Pittman, S Spector. 1992. Analytical methods validation: bioavailability, bioequivalence and pharmacokinetic studies. J Pharm Sci 81, 309-312

Shoop W, H Mrozik, M Fisher. 1995. Structure and activity of avermectins and milbemycins in animal healt. Vet Parasitol. 59, 139-156..

Slanina P, J Kuivinen, CH Ohlsén, L-G Ekstrom. 1989. Ivermectin residues in the edible tissues of swine and cattle: effect of cooking and toxicological evaluation. Food Addit \& Contam 6, 475-481.

Toutain PL, M Campan, P Galtier, M Alvinerie. 1988. Kinetic and insecticidal propieties of ivermectin residues in the milk of dairy cows. J Vet Pharmacol Therap 11, 288-291. 